\title{
CAPITULO21
}

\section{ANÁLISE DO PERFIL EPIDEMIOLÓGICO DOS CASOS DE HEPATITES VIRAIS NO NORDESTE DE 1999 a 2019}

DOI 10.4322/978-65-995353-2-1.c21

\begin{abstract}
Thiemmy de Souza Almeida Guedes ${ }^{1}$, Caroline Cristina de Oliveira Cunha ${ }^{2}$, Fernanda Beatriz Freitas dos Santos ${ }^{3}$, Larissa Lima Soares ${ }^{4}$, Livia Maria Tavares Miranda ${ }^{5}$, Rebeca Margarida Silva Santana dos Santos ${ }^{6}$, Angélica Jesus Rodrigues Campos ${ }^{7}$, Caroline Taiane Santos da Silva ${ }^{8}$

${ }^{1}$ Faculdade Venda Nova do Imigrante, (thiemmyalmeida@gmail.com) 2Escola Superior Madre Celeste, (caroline.ccunha@gmail.com) 3Universidade Salvador, (ffernandabfreitas@gmail.com) ${ }^{4}$ Faculdade Venda Nova do Imigrante, (larissalyma@hotmail.com) ${ }^{5}$ Faculdade Venda Nova do Imigrante, (livia.miranda12@gmail.com)

${ }^{6}$ Universidade Salvador, (rebecamagsantos@ hotmail.com)

${ }^{7}$ Universidade Federal do Piauí, (angelicajesus@ufpi.edu.br)

${ }^{8}$ Child Behavior Institute of Miami, (carolinetaiane.enfa@gmail.com)
\end{abstract}

\section{Resumo:}

Objetivo: Analisar o perfil epidemiológico dos casos de Hepatites Virais no Nordeste entre os anos de 1999 a 2019. Método: Trata-se de um estudo epidemiológico de caráter descritivo e quantitativo, que visa relacionar as informações de domínio público disponibilizadas através da plataforma DATASUS. Para coleta de dados, o estudo selecionará as variáveis de todos os municípios brasileiros do Nordeste como região, sexo, classificação da hepatite, faixa etária e taxa de óbito; posteriormente os resultados serão dispostos em tabelas com a ajuda do programa Microsoft Excel. Resultados: Após a análise dos dados, verificou-se que o Brasil apresentou um total de 92.582 casos notificados de Hepatites Virais (HV), entre os anos de 1999 a 2019, dentre os quais a Hepatite A, obteve um maior número de notificações, com 50.567 registros; quanto aos números de óbitos, a Hepatite $\mathrm{C}$ teve 16.382 registros; em relação ao sexo que registrou maior notificação, com 26.653 casos, foi o masculino; em relação as notificações por região, a Região Sudeste foi a que mais obteve registros, com 272.528 casos, dos quais a Hepatite $\mathrm{C}$ é a que mais foi notificada; o estado do Nordeste com grande número de registros foi a Bahia com 23.519 notificações, tendo como prevalente a Hepatite A. Conclusões: Diante o exposto, pode-se inferir que atitudes relacionadas à promoção da saúde da população e programas de saneamento básico em conjunto com a vacinação adequada disponível evitaria 
números crescentes quanto à contaminação de Hepatites Virais.

Palavras-chave: Hepatite Viral Humana; Epidemiologia; Sistema de Informações em Saúde. Área Temática: Ciências da Saúde.

E-mail do autor principal: thiemmyalmeida@gmail.com

\section{INTRODUÇÃO}

As doenças causadas por vírus, particularmente as Infecções Sexualmente Transmissíveis (IST), constituem um grave problema de saúde pública a nível mundial devido a sua transmissibilidade. Por isso, uma das doenças que se tornou um dos Objetivos de Desenvolvimento Sustentável (ODS) da Organização das Nações Unidas (ONU) foram as hepatites. As Hepatites virais, que são geradas por diferentes agentes etiológicos e desenvolvem-se principalmente no fígado, apresentam características clínicas, epidemiológicas e laboratoriais características. Sendo assim, a ONU objetivou a ODS com a finalidade da diminuição do contágio e eliminação da mortalidade relacionada a esta doença.

Estudos apontam que os vírus que mais causam hepatites são: A, B, C, D e E; dos quais os do tipo B, C e D são considerados mais graves pela possibilidade de tornarem-se crônicos (BRASIL, 2005). De acordo com o Ministério da Saúde (2005), as hepatites virais são doenças de notificação compulsória (DNC) e passaram a ser notificadas a partir de 8 de dezembro de 2003; estas notificações ocorrem com o preenchimento de fichas específicas para hepatites virais e demais doenças compulsórias no Sistema Nacional de Agravos e Notificação (SINAN) após investigação e detecção pelos centros de saúde responsáveis (BRASIL, Portaria de $n^{\circ}$ 2529, de 23 de novembro de 2004).

Um dos aliados para o enfrentamento das hepatites é o Sistema Único de Saúde (SUS), que conta com o Programa Nacional para a Prevenção e o Controle das Hepatites Virais (PNHV), que foi criado pelo Ministério da Saúde em 5 de fevereiro de 2002, visando ações que contribuem para o aprimoramento de intervenções em saúde relacionadas às hepatites; e, com isso, houve a criação de várias políticas públicas de saúde voltadas a prevenção e promoção.

Conforme dados do Boletim Epidemiológico das Hepatites Virais de 2020, de 1999 a 2019, foram notificados no Sistema de Informação de Agravos de Notificação (Sinan) 673.389 casos confirmados de hepatites virais no Brasil. Dados tão significativos levaram a Organização Mundial da Saúde (OMS) a assumir, como um dos seus objetivos, eliminar as hepatites virais até 2030. A partir dessas considerações e dada a importância de estudos epidemiológicos acerca das hepatites virais, esta pesquisa vem analisar o perfil epidemiológico

\section{E - book Tripé do Ensino Superior: Ensino,} Pesquisa e Extensão 
dos casos no Nordeste entre os anos de 1999 e 2019.

\section{MÉTODO}

Trata-se de um estudo epidemiológico de caráter descritivo e quantitativo, que visa relacionar as informações de domínio público disponibilizadas através da plataforma DATASUS. Segundo Lima-Costa \& Barreto (2003) o estudo da epidemiologia é caracterizado através da distribuição e determinantes de enfermidades ou circunstâncias relacionadas à saúde de uma população específica. Sendo assim, esse tipo de pesquisa inclui: vigilância, observação e experimento. Para Knechtel (2014) a pesquisa na modalidade quantitativa, aborda sobre um problema que pode ser humano ou da sociedade, é um estudo com base em variáveis quantificadas, sendo assim, o objetivo é determinar as análises estatísticas.

Para coleta de dados, o presente estudo se propôs a analisar os dados epidemiológicos registrados no Sistema de Indicadores e Dados Básicos das hepatites nos municípios brasileiros nos anos de 1999 a 2019. As variáveis do estudo dizem respeito a região, sexo, classificação da hepatite, faixa etária e taxa de óbito. Os dados então, serão analisados e quantificados em tabelas, utilizando o Microsoft Excel ${ }^{\mathrm{TM}}$ versão 2016.

Por se tratar de um banco de dados de domínio público, não foi necessária submissão ao comitê de ética. De acordo com os termos da Lei n ${ }^{\circ} 12.527$ de 18 de novembro de 2011 que fundamenta pesquisas com informações de livre acesso (BRASIL, 2011).

\section{RESULTADOS E DISCUSSÃO}

Na tabela 1 pode-se verificar, de acordo com o Ministério da Saúde, que o Brasil apresentou um total de 92.582 casos notificados de Hepatites Virais (HV), entre os anos de 1999 a 2019, dentre os quais a Hepatite A, obteve um maior número de notificações, com 50.567 registros, seguindo da Hepatite B com 25.313, Hepatite C com 16.382, e Hepatite D com 229.

As regiões Norte e Nordeste apresentaram 55,7\% de todos os casos de Hepatite A durante os anos de 1999 a 2018. A doença tem grande relação com alimentos ou água não seguros, baixos níveis de saneamento básico e de higiene pessoal (OMS, 2019). Segundo o Sistema Nacional de Informações sobre Saneamento (SNIS), em 2019, apenas 28,9\% da população do Nordeste tinha atendimento de esgotos. Esse déficit de mais de $50 \%$ da população nordestina sem a cobertura devida, aumenta a probabilidade de contaminação com inúmeros patógenos prejudiciais à saúde, incluindo a Hepatite A (HV A), onde a transmissão se dá de forma fecal-oral, isto é, partir do contato de fezes com a boca. 
De acordo com os dados coletados, no período de 1999 a 2019 foram notificados um total de 92.582 casos de hepatites virais no Brasil, dentre os quais 50.657 pertenciam a HV A, seguindo HV B com 25.313 casos, HV C com 16.382 e HV D com 229 registros de casos. O vírus da hepatite A é o causador mais comum da hepatite viral aguda do mundo. De acordo com uma hipótese realizada pela Organização Pan-americana de Saúde, por ano acontecem no Brasil aproximadamente 130 novos casos de HVA, por 100.000 pessoas. Atualmente, continua sendo um grande desafio de saúde pública no Brasil, e o país é considerado uma área de risco para esta doença (MINISTÉRIO DA SAÚDE, 2021).

Tabela 1 - Total de casos notificados entre 1999 a 2019 no Brasil.

\begin{tabular}{|c|c|c|}
\hline Casos de hepatites virais & $\mathbf{N}$ & \% \\
\hline A & 50.657 & 54,71 \\
\hline B & 25.313 & 25,67 \\
\hline C & 16.382 & 17,69 \\
\hline D & 229 & 0,24 \\
\hline Total & 92.582 & 100 \\
\hline
\end{tabular}

Fonte: MS, 2021.

Nota-se que na tabela 2, há um maior número de óbitos por hepatite $\mathrm{C}$, equivalente a $61,12 \%$ do total de casos, seguida pela hepatite B com 26,18\%. Em comparação, pode-se afirmar que esses dados corroboram com os dados mundiais da Organização Mundial da Saúde (OMS), que revela que as hepatites virais são responsáveis por mais de 1,34 milhão de óbitos anualmente, dos quais 66\% são causadas pela hepatite B e 30\% pela hepatite C (OMS, 2017), revelando assim, que estes tipos de hepatites são os mais letais.

A hepatite C, por sua vez, caracteriza-se por um processo infeccioso e inflamatório, e que pode se manifestar na forma aguda ou crônica, sendo esta segunda a forma mais comum, sendo 60\% a 85\% dos casos (MINISTÉRIO DA SAÚDE, 2021). O Ministério da Saúde, desde 1989, dispõe da vacina da HVB, e desde o ano de 2014 da HVA, as quais proporcionam coberturas vacinais das hepatites B e A. Entretanto, para a hepatite do tipo C não existe uma vacina, por isso, em estudo realizado por CAMPOS (2019), o autor faz uma relação entre este fator com o grande número de infecções e óbitos notificados.

\section{E - book Tripé do Ensino Superior: Ensino,} Pesquisa e Extensão 
Tabela 2 - Relação entre Classificação da Hepatite e total de óbitos entre 1999-2019.

\begin{tabular}{|c|l|l|}
\hline $\begin{array}{c}\text { CLASSIFICAÇÃO DAS } \\
\text { HEPATITES VIRAIS }\end{array}$ & \multicolumn{1}{|c|}{ N } & \multicolumn{1}{|c}{} \\
\hline A & 302 & 5,82 \\
\hline B & 1.357 & 26,18 \\
\hline C & 3.479 & 61,12 \\
\hline D & 45 & 0,86 \\
\hline Total & 5.183 & 100 \\
\hline
\end{tabular}

Fonte: MS, 2021.

Já na tabela 3, pode-se observar que há uma prevalência de maior contaminação em pacientes do sexo masculino. E isto pode ser justificado pelo fato de haver, no caso dos homens, uma exposição a diferentes fatores de riscos, como por exemplo: a não utilização de preservativos durante $\mathrm{o}$ ato sexual, uma maior quantidade e rodízio de parceiras(os), considerando que estas estão totalmente relacionadas à transmissão de vírus de HV(DIAS, 2014).

Tabela 3 - Relação entre Classificação da Hepatite e sexo entre 1999-2019.

\begin{tabular}{c|c|c|c|c}
\hline \multirow{2}{*}{$\begin{array}{c}\text { CLASSIFIC } \\
\text { AÇÃO DA }\end{array}$} & \multicolumn{2}{|c|}{ FEMININO } & \multicolumn{2}{c}{ MASCULINO } \\
\cline { 2 - 5 } HEPATITE & N & $\%$ & N & \\
& & & & \\
A & 23.980 & 47,36 & 26.653 & 52,63 \\
\hline B & 11.672 & 46,13 & 13.629 & 53,86 \\
\hline C & 6.861 & 41,89 & 9.516 & 58,10 \\
\hline \multirow{2}{*}{ D } & 102 & 44,54 & 127 & 55,45 \\
\hline
\end{tabular}


Fonte: MS, 2021.

Em relação aos números de notificações por região e a classificação das $\mathrm{HV}$, observou-se na Tabela 4 que a Região Sudeste foi a que mais obteve registros, com 272.528 casos, dos quais a Hepatite C é a que mais foi notificada. A Região Sudeste foi seguida pela Região Sul com 179.347 registros, Região Nordeste com 94.163, Região Norte com 92.273, e por último a Região Centro-Oeste com 51.320 casos registrados. Observa-se que nas regiões Sul e Sudeste, ficou evidenciado um maior número de casos diagnosticados, e, dessa forma, possivelmente o sistema de vigilância epidemiológica tenha uma melhor qualidade (NUNES et al, 2017).

Tabela 4 - Relação de número notificados por região e classificação de Hepatites Virais 1999-2019.

\begin{tabular}{|c|c|c|c|c|c|}
\hline $\begin{array}{c}\text { REGIÃO } \\
\text { HEPATITE }\end{array}$ & NORDESTE & NORTE & SUL & SUDESTE & $\begin{array}{c}\text { CENTRO- } \\
\text { OESTE }\end{array}$ \\
\hline A & 50.727 & 42.655 & 26.013 & 30.364 & 18.716 \\
\hline B & 26.140 & 37.233 & 80.902 & 87.044 & 22.903 \\
\hline C & 17.078 & 9.288 & 72.171 & 154.690 & 9.568 \\
\hline D & 218 & 3.107 & 261 & 430 & 133 \\
\hline Total & 94.163 & 92.273 & 179.347 & 272.528 & 51.320 \\
\hline
\end{tabular}

Fonte:

MS,

2021.

Analisando a tabela 5, pode-se verificar que o estado da região Nordeste que mais teve casos registrados de hepatites virais foi a Bahia, contudo, o estado de Pernambuco foi o que apresentou aumento no número de notificação de Hepatite A; os registros realizados por outros tipos de HV também teve maior incidência no estado da Bahia. Estudos relatam que um grande fator de risco associado, a contaminação pelo vírus hepatite A, é a estrutura do saneamento básico ou até mesmo a falta, visto que a transmissão se dá através da ingestão de água ou alimentos contaminados com matéria fecal (MARTINS, 2020); uma realidade 
presente

em quase

todas

as

cidades

do

Brasil.

Tabela 5 - Casos de Hepatites Virais por estados da região Nordeste entre 1999-2019.

\begin{tabular}{|c|c|c|c|c|c|}
\hline $\begin{array}{c}\text { CASOS DE } \\
\text { HEPATITES } \\
\text { VIRAIS POR } \\
\text { ESTADOS }\end{array}$ & HEP. A & HEP. B & HEP. C & HEP. D & TOTAL \\
\hline ALAGOAS & 4.028 & 2.366 & 1.123 & 18 & 7.535 \\
\hline BAHIA & 9.285 & 7.773 & 6.419 & 42 & 23.519 \\
\hline CEARÁ & 6.687 & 3.127 & 2.203 & 25 & 12.042 \\
\hline MARANHÃO & 6.840 & 3.694 & 1.436 & 41 & 12.011 \\
\hline PARAÍBA & 5.088 & 1.842 & 900 & 14 & 7.844 \\
\hline PERNAMBUCO & 10.894 & 3.704 & 2.295 & 51 & 16.944 \\
\hline PIAUÍ & 3.764 & 757 & 479 & 12 & 5.012 \\
\hline R. G. NORTE & 2.657 & 888 & 1.076 & 8 & 4.629 \\
\hline SERGIPE & 1.484 & 1.989 & 1.147 & 7 & 4.627 \\
\hline
\end{tabular}

Fonte: MS, 2021.

\section{CONCLUSÃO}

Diante o exposto, pode-se inferir que atitudes relacionadas à promoção da saúde da população e programas de saneamento básico em conjunto com a vacinação adequada disponível evitaria números crescentes quanto à contaminação de Hepatites Virais.

Dessa forma, conclui-se que se faz relevante a criação de um maior arcabouço teórico sobre a temática com foco na incidência dos casos de hepatites virais na região nordeste, para subsidiar órgãos da vigilância sanitária a detectarem informações sobre prevenção, diagnóstico, tratamento e controle da transmissão da doença. É necessário também atentar-se à subnotificação dos casos, pois, por conta disso, podem ser encontradas incoerências entre dados epidemiológicos registrados e a literatura científica, impossibilitando ações de prevenção em saúde. 


\section{REFERÊNCIAS}

BRASIL. Ministério da Saúde. Doenças infecciosas e parasitárias: guia de bolso. Ministério da Saúde, Secretaria de Vigilância em Saúde. Brasília: Ministério da Saúde, 2005.

BRASIL. Ministério da Saúde. Portaria no 2529, de 23 de novembro de 2004. Institui o Subsistema Nacional de Vigilância Epidemiológica em Âmbito Hospitalar, define competências para os estabelecimentos hospitalares, a União, os estados, o Distrito Federal e os municípios, cria a Rede Nacional de Hospitais de Referência para o referido Subsistema e define critérios para qualificação de estabelecimentos. Brasília: Ministério da Saúde, 2004.

BRASIL. Ministério da Saúde. Programa Nacional de Hepatites Virais. Avaliação da Assistência às Hepatites Virais no Brasil. Brasília: Ministério da Saúde, 2002.

CAMPOS, Jessica Freire. et al. Hepatite C: os avanços para o tratamento da doença baseado em pedidos de patentes da vacina e medicamentos contra o vírus. Revista INGI - Indicação Geográfica e Inovação, v. 3, n. 4, p. 540-553, 2019. Disponível em: < http://www.ingi.api.org.br/index.php/INGI/article/view/70>. Acesso em 20 ago. 2021.

DIAS, Jerusa Araújo; CERUTTI, Crispim; FALQUETO, Aloísio. Fatores associados à infecção pelo vírus da hepatite B: um estudo caso-controle no município de São Mateus, Espírito Santo. Epidemiologia e Serviços de Saúde, v. 23, n. 4, p. 683-690, 2014. Disponível em: <https://doi.org/10.5123/S1679-49742014000400010>. Acesso em: 20 ago. 2021.

KNECHTEL, Maria do Rosário. Metodologia da pesquisa em educação: uma abordagem teórico-prática dialogada. Curitiba: Intersaberes, 2014.

LIMA-COSTA, Maria Fernanda; BARRETO, Sandhi Maria. Tipos de estudos epidemiológicos: conceitos básicos e aplicações na área do envelhecimento. Epidemiol. Serv. Saúde, v. 12, n. 4, p. 189-201, 2003. Disponível em: <http://dx.doi.org/10.5123/S167949742003000400003>. Acesso em: 20 ago. 2021.

MARTINS, F. S.; POTSCH, D.V.; GASPAR, A. M. Hepatite A: Cuidados com a higiene e o Saneamento Básico. Boa Saúde, 2004. Disponível em: <https://www.boasaude.com.br/artigosde-saude/3973/-1/hepatite-a-cuidados-com-a-higienee-o-saneamento-basico.html>. Acesso em: 23 ago 2021.

NUNES, Heloisa Marceliano. et al. As hepatites virais: aspectos epidemiológicos, clínicos e de prevenção em municípios da Microrregião de Parauapebas, sudeste do estado do Pará, Brasil. Revista Pan-Amazônica de Saúde, v. 8, n. 2, p. 29-35, 2017. Disponível em: <https://dx.doi.org/10.5123/s2176-62232017000200004>. Acesso em: 20 ago. 2021.

OMS, Organização Mundial da Saúde. Global hepatitis report, 2017. Disponível em: <https://www.who.int/hepatitis/publications/global-hepatitis-report2017/en>. Acesso em: 20 ago. 2021. 